\title{
Sign-Based Semantics-Enriched Social Computing
}

\author{
Jingzhi Guo \\ Department of Computer and Information Science, University of Macau \\ Av. Padre Tomás, Pereira, S.J., Taipa, Macau, Tel: +853-83974360 \\ E-mail:jzguo@umac.mo
}

\begin{abstract}
During the transformation of the existing social Web to semantic social Web, semantics-enriched social computing is becoming important, because it can support semanticsoriented user connection and collaboration in a larger community. To provide this support, this paper proposes a novel sign-based social computing approach, called Sign Network, to represent and exchange semantically interoperable Web content between online computer systems. This approach semantically enriches social content and enables to mediate the communication of Social Web users such that their social content can be unambiguously created and used. Applying this approach, a social sign exchange mechanism is developed for enabling the applications of social tagging, social navigation and social collaboration.
\end{abstract}

\section{Introduction}

Internet is experiencing a drastic change when it is transformed from the traditional Internet to socially-connected Web. In this transformation, the intensive user participation of social Web asks for the research of social computing [25] to analyze, study, and support connectivity, collaboration and community of Web users [15]. It also requires the incorporation of semantics study [20]. Semantics can be defined as the machine-computable, human-understandable, and program-reasonable meanings of concepts for the present and future Web. It is one of the most important tasks of social computing, which has to be fulfilled in order to enable efficient connection, better collaboration and larger community for Web evolution.

Classically, social computing "describes any type of computing application in which software serves as an intermediary or a focus for a social relation" [21] in the aspects such as communication, governance, education, privacy, collaboration and decision-making. Modern definition adds research elements of interaction, social structure, social study, human-social dynamics, and social context [25]. From these definitions, the commonality is what ties Web users together and how they are tied in the context of computing network. The former emphasizes on why Web users are coupled together - whether they have dependences of goal, resources, tasks, functions, activities, constraints, rules of conduct, intentions, preferences, information sharing, and communication networks [7], whether they have fun in a social entity [4] (e.g. worldofwarcraft.com, qq.com), or whether they have social capital for access (e.g. Wikipedia.com). The latter focuses on computing technology that could support the coupling of Web users - in what networking techniques enable Web users to connect and collaborate together in a social entity. Obviously, "why coupling together" is more associated to the studies such as psychology, sociology, anthropology, management, economics, ethnics and political science, while "how coupling together" is in the technical domain of computing science yet linking to the changing Web. The former provides the requirement specifications to the latter, while the latter is the design and implementation of the former.

Social computing has many applications [25], such as online communities (e.g., blogs, wikis, social networks, collaborative bookmarking, social tagging, and podcasts), business and public sector (e.g., e-marketplace such as alibaba.com or amazon.com, virtual marketplace such as xstreetsl.com, e-government such as igov.gov.sg), interactive entertainment (e.g., game zones such as game.qq.com and worldofwarcraft.com), and virtual world (e.g. secondlife.com). To enable these applications, many computing technologies are needed, which are technologies of Web, database, multimedia, mobile computing, multi-agents, and software engineering. Currently, key research issues in social computing are representation of social information and knowledge, agent-based social modeling, and analysis and prediction of social phenomena [25].

In the above key research issues, one of most difficult problems is how to semantically represent social information and knowledge, for example, social tagging for social navigation and social collaboration. An appropriate solution to this problem directly affects whether the present Social Web can be successfully transformed into the future Semantic Social Web [20]. Semantic representation refers to a computing technique of depicting the meanings given by users for their provided contents. It targets at building a semantic mapping relationship between the domain of user content and a domain of socially computable and understandable sign set without any meaning ambiguity and inconsistency [11][14].

Semantic representation is very important to social computing. This is because users of Social Web are creative and have individual perspectives due to diverse backgrounds of 
natural languages, cultures, customs and behaviors [6]. Technically speaking, users in interaction with diverse backgrounds are contextual, which means that their contents are autonomous, distributed, emergent yet interdependent each is similar to a "meaning island" that cannot meaningfully communicate with each other. These properties of Social Web make the meaningful interaction between Web users extremely difficult and lead to ambiguities in content understanding between Web users. These imply that the domain of user content cannot be effectively mapped onto the domain of socially computable and understandable sign set, where meaning interoperability is assumed. The noninteroperability of meaningful contents severely affects the connectivity and collaboration between Social Web users and reduces the opportunity of social community formation. This further harms the healthy evolution of Social Web to Semantic Social Web.

This paper aims to propose a novel sign-based social computing approach, called Sign Network, to solve the problem of meaning interoperation between the contents of Social Web users. This approach semantically enriches social content and enables to mediate the communication of Social Web users such that their created social content can be unambiguously created and used for social tagging, navigation and user collaboration.

The rest of this paper is organized as follows. Section 2 makes a review of existing content representation methods. Section 3 introduces a new sign network. Section 4 describes the sign-based social computing mechanism for social computing. Section 5 makes a comparison between ontology and sign. Finally, a conclusion is made and the contribution, limitation and future work are discussed.

\section{Related Work}

One of the important tasks of social computing is to provide a medium to support meaningful communication between Web users. This particularly reflects in the requirement for a semantic representation system, which enables to mediate social tagging, social navigation and user collaboration in a semantic consistent way. Many existing researches targets at this mediation function by developing particular solutions to semantic representations.

One common solution to adding semantics to content is to build a collaborative tool, where people can collaboratively represent content semantics, in terms of annotations or tags. TagSEA [23] is an example, which provided automatic tagging of source codes for distributed team members to use. Similar examples can be seen in Dogear [16].

The second solution attempts to find semantic associations between tags and thus to build a classification of meaningful tags for Web users to deploy. CubeLSI [3] is an approach that proposed a Cube Latent Semantic Indexing technique through representing a data structure as a thirdorder tensor to capture latent semantic associations between tags, such that the correlations between users, tags and re- sources can be captured simultaneously. Many similar semantic techniques can be found. For example, SocialTagger [24] used the multivariate statistical technique of canonical correlation analysis to associate blogs and tags and thus find their semantic relations. Other examples employing associations and similarities could be [13][19][26].

The third solution employs Wikipedia as tag space to mediate heterogeneous concepts. For example, [22] proposed to apply Wikipedia metadata as a source of mapping onto different resources and Web pages. Similar suggestion can be found in [12].

For the above approaches, the first approach merely provides a shared view for other users to check and action with minimal machine understanding. The second approach is largely automated to find semantics convergence with classification, association and clustering techniques. The third approach often applies Wiki tags as a controlled vocabulary to share semantics. This approach is also often used in the first and second approaches. Though all these approaches have merits to a certain degree of helping semantics disambiguation between contextually different Web users, they can only alleviate the severeness of the problem. This is because Solution 1 suggests that "what I tag is your tag", so the other tag users still need to make correct interpretation of the tag meaning. This solution is limited in a contextually similar environment. Solution 2 cannot avoid the similarity problem, which assumes that similarity is enough for content interpretation. Nevertheless, similarity solution cannot build applications that require $100 \%$ accuracy (e.g. interpreting a payment bill). For Solution 3, it always cannot prevent the different interpretation of Wiki tags unless Wiki tags become a semantically consistent vocabulary between all dictionaries that Web users are implicitly using. However, it seems mission-impossible.

\section{Sign-based Semantic Representation}

Adding accurate semantics, but not semantic similarity, to social computing relates to the research of semantic representation. To semantically enrich social computing, this paper introduces a sign-based semantic representation for the existing social computing technology.

\subsection{Concept of Sign}

In general, existing semantic representation research is stemmed from two studies: the science of being originated from Aristotle [1] in philosophy, and the theory of sign driven by Saussure [18] and Peirce [17] in semiotics. These two schools of study gradually become the theoretical foundation of semantic representation in computer science. The former is now popularly known as ontology [9] and the latter is simply called as sign [11][14]. Both are a type of semantic representation, attempting to computationally depict the outcome of our living world for computing purpose. The basic definition of ontology is "an explicit specification of a conceptualization" [9] of things. It is a formal represen- 
tation of the objective concept in reference to any things for computers to read within a shared domain. Slightly different, the sign in computer science defines the relations between a real-world object, its interpretation and the interpreted sign referring to the object, regardless of whether this referred object is physical, conceptual or fictitious. Any sign is an interpretational representation of the subjective concept in reference to the referred object for computers to process and mediate human communicating meanings across multiple discrete domains [8][11].

\subsection{Semiotic Sign as Theoretical Foundation of Representing Social Content}

This paper adopts semiotic sign as a means of semantic representation. Semiotics thinks that anything in reality that is represented is a sign. We think that anything digitally represented in computing systems is a sign. A sign can be a bit of zero and one, a byte of octet, an integer, a string, a graph, a text, or a piece of code, etc.

As the theoretical foundation of social content representation in this paper, semiotic sign is particularly influenced by the dyadic sign model of Saussure [18], the triadic sign model of Peirce [17], and orders of signification on signs of Bathes [2]. Saussure thinks that a sign is dyadic and consists of a signifier (the structure of sign) and a signified (the concept of a sign). Peirce thinks that a sign is triadic, where real-world object is interpreted by interpretant as sign, which again refers to that object. Bathes thinks that for any signifier and signified of a sign, a signifier again becomes a sign when signified. This recursive process leads to a chain of signification.

Sign Network proposed in this paper combines these theories together to form a new computational sign theory by adding context. Its perspective can be shown in Figure 1, which explains how the meaning of a sign comes from, and why a sign should be designed so.

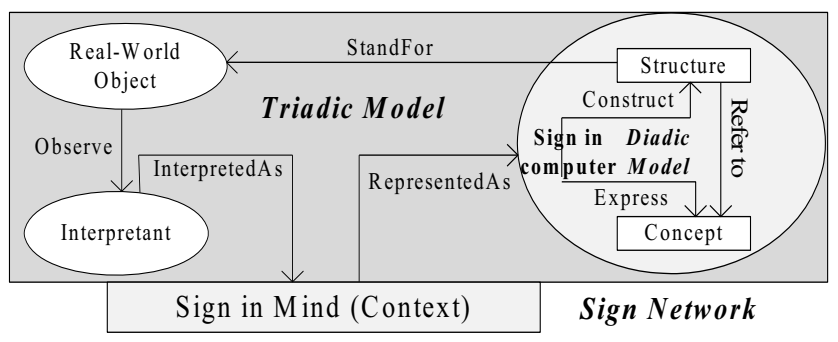

Figure 1: A Sign-based View to Concept Representation

In Sign Network, we think dyadic model perfectly reflects the relation between sign structure and sign meaning (i.e. concept). But it lacks interpretation. We thus place dyadic sign in triadic model (i.e. object, interpretant, sign) where a sign is an interpretation result of the real world object. However, a simple tradic model is still not enough because different interpretations happen in heterogeneous contexts. Thus, Sign Network adds a context between Peirce's interpretant and Saussure's sign, where a context is an individual interpretation of similar real-world objects as a mind sign that can again be represented as a sign in computer. Although there many definitions about context (e.g. see related discussion in [10]), context is pragmatic and specifies "the relationships between signs and their users" [5]. It is a perspective of sign building comparing with the other perspectives of building signs.

\subsection{Sign Network}

Applying the semiotic sign theory, we design a Sign Network based on the generic concept exchange model developed in collaborative conceptualization approach [10][11]. The proposed Sign Network can be described in Figure 2.

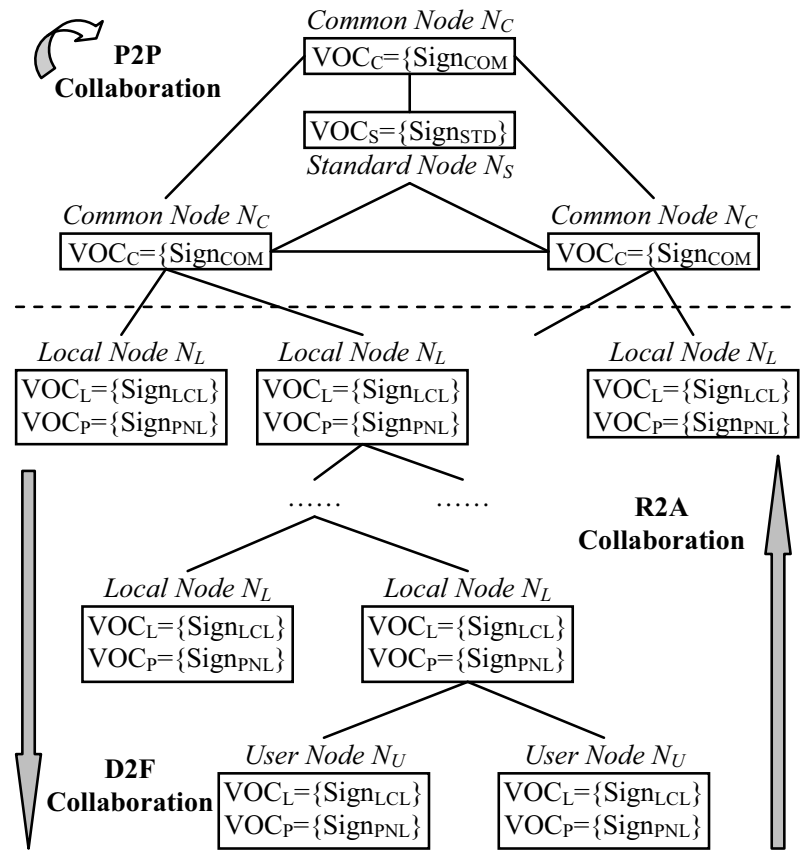

Figure 2: Sign Network

In the above Sign Network, there are four categories of signs: standard signs ( sign $_{\mathrm{STD}}$ ) that form a standard vocabulary $\left(\mathrm{VOC}_{\mathrm{S}}\right)$ located in a standard node $\left(\mathrm{N}_{\mathrm{S}}\right)$, common signs ( sign $\left._{\mathrm{COM}}\right)$ forming different common vocabularies $\left(\mathrm{VOC}_{\mathrm{C}}\right)$ in common nodes $\left(\mathrm{N}_{\mathrm{C}}\right)$, local signs $\left(\operatorname{sign}_{\mathrm{LCL}}\right)$ composing a variety of local vocabularies $\left(\mathrm{VOC}_{\mathrm{L}}\right)$ in local nodes $\left(\mathrm{N}_{\mathrm{L}}\right)$, and personal signs $\left(\operatorname{sign}_{\mathrm{PNL}}\right)$ creating many personal vocabularies $\left(\mathrm{VOC}_{\mathrm{P}}\right)$ in local nodes $\left(\mathrm{N}_{\mathrm{L}}\right)$ and user nodes $\left(\mathrm{N}_{\mathrm{U}}\right)$.

The basic presentation of each sign, extended from Product Map representation [11], is defined as follows,:

$$
\begin{gathered}
\text { Sign }=(\mathrm{S}, \mathrm{C}, \mathrm{X}, \diamond) \\
\mathrm{S}(\text { Sign })=\mathrm{S}(\mathrm{MD}-\mathrm{IID}, \mathrm{T}, \mathrm{AN}, \mathrm{X},<\mathrm{OP}>) \\
\mathrm{C}(\mathrm{Sign})=\mathrm{C}(\mathrm{MD}-\mathrm{IID} \equiv \mathrm{T} \Leftarrow \mathrm{AN} @ \mathrm{X}<<\diamond)
\end{gathered}
$$

where a sign is a tuple of structure $(\mathrm{S})$, concept $(\mathrm{C})$, context $(\mathrm{X})$ and interpretant $(\diamond)$ as defined in (1.1). The structure of a sign is a tuple of internal identifier (MD-IID), term (T), annotation (AN), context (X) and some optional elementary 
structures $(<\mathrm{OP}>)$ as defined in (1.2). The concept of a sign is conveyed through a set of defined relations as defined in (1.3), such that a concept annotation (AN) of a sign (i.e. the sense or meaning of a sign), which is interpreted by an interpretant $\diamond$ at the context $\mathrm{X}$, determines the sense of a synonymous term (T) uniquely identified by an identifier (IID).

\subsubsection{Term Sense Disambiguation}

For the categorized signs, standard sign designers in standard node create and modify standard signs; common sign designers of common nodes collaboratively design common signs in a peer-to-peer (P2P) mode; local sign designers in each local node localize common signs into local signs in a dominator-to-follower (D2F) mode; sign designers in local and user nodes also create their personal signs in their own ways.

Particularly, in designing standard signs and common signs, term sense disambiguation (TSD) work is important and done by P2P collaborators and arbitrator (a final decision-maker for term sense disambiguation). The TSD work includes further identifying the sense scope of T:

$$
\mathrm{T}=(\mathrm{ct}, \mathrm{ctx}, \mathrm{fcx}, \mathrm{smp})
$$

where "ct" denotes part of speech, "ctx" denotes general term relations, "fcx" denotes ConexNet term relations, and "smp" denotes term samples, such that:

$$
\mathrm{ct}=(n, v, a, r, m, q, p, o, e, d, h, g, s)
$$

where $n$ is noun, $v$ is verb, $a$ is adjective, $r$ is adverb, $m$ is numeral, $q$ is quantifier, $p$ is proposition, $o$ is pronoun, $e$ is exclamation, $x$ is onomatopoeia, $d$ is determinant, $h$ is fixed phrase, $g$ is math formula that can be parsed in a fixed way by computer (math formula is a separate study), and $s$ is symbol used in any computer systems and created by sign network.

In Sign Network, any term $t \in \mathrm{T}$ is defined is in a Sign Network grammar tree, shown in Figure 3 (as an example).

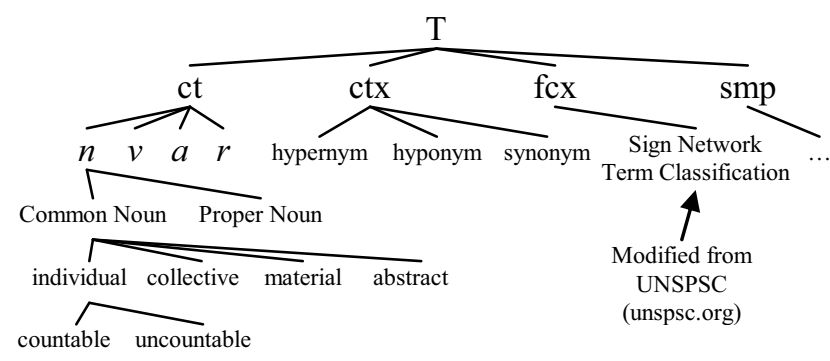

Figure 3: Sign Network Term Grammar Tree

This tree is used to disambiguate a term to a clear meaning for a sign along with its sense $\mathrm{AN}$ interpreted by an interpretant $\diamond$.

\subsubsection{Independence of Unique Sign Identification}

A sign in Sign Network requires unique identifier without causing any sign versioning problem. This requires that each sign used to construct vocabularies must be atomic and independent of each other. To solve this problem, this paper introduces a novel multi-dimensional Internal Identifier Scheme (MD-IID Scheme) such that MD-IID = $\mathrm{NS}_{\mathrm{x}}$ :OT-CT::NS $\mathrm{y}$ :OC-CT (see Table 1), where $\mathrm{NS}_{\mathrm{x}}$ and $\mathrm{NS}_{\mathrm{y}}$ are any two 3-dimensional sign spaces such that NS = $\mathrm{X}$ ( $\mathrm{X}$ is node address in Def. 1.3), OT and CT are original and current timestamps for identifying concept $\mathrm{C} @ \mathrm{NS}$ at creation time and current time of a sign. This scheme guarantees each sign used in a vocabulary is atomic and independent (the proof will be discussed elsewhere).

Table 1: MD-IID Scheme

\begin{tabular}{|c|l|l|}
\hline Form & \multicolumn{1}{|c|}{ Meaning } & \multicolumn{1}{c|}{ Notation } \\
\hline 1 & Original sign in $\mathrm{NS}_{\mathrm{x}}$ & MD-IID $=\mathrm{NS}_{\mathrm{x}}: \mathrm{OT}-\mathrm{OT}$ \\
\hline 2 & Modified sign in $\mathrm{NS}_{\mathrm{x}}$ & MD-IID $=\mathrm{NS}_{\mathrm{x}}: \mathrm{OT}-\mathrm{CT}$ \\
\hline 3 & $\begin{array}{l}\text { Original interpretation of original } \\
\text { sign of } \mathrm{NS}_{\mathrm{x}} \text { in } \mathrm{NS}_{\mathrm{y}}\end{array}$ & $\begin{array}{l}\text { MD-IID }=\mathrm{NS}_{\mathrm{x}}: \mathrm{OT}-\mathrm{OT}:: \\
\text { NS }: \text { OT-OT }\end{array}$ \\
\hline 4 & $\begin{array}{l}\text { Original interpretation of modified } \\
\text { sign of } \mathrm{NS}_{\mathrm{x}} \text { in } \mathrm{NS}_{\mathrm{y}}\end{array}$ & $\begin{array}{l}\text { MD-IID }=\mathrm{NS}_{\mathrm{x}}: \mathrm{OT}-\mathrm{CT}:: \\
\mathrm{NS}_{\mathrm{y}}: \mathrm{OT}-\mathrm{OT}\end{array}$ \\
\hline 5 & $\begin{array}{l}\text { Modified interpretation of original } \\
\text { sign of } \mathrm{NS}_{\mathrm{x}} \text { in } \mathrm{NS}_{\mathrm{y}}\end{array}$ & $\begin{array}{l}\text { MD-IID }=\mathrm{NS}_{\mathrm{x}}: \mathrm{OT}-\mathrm{OT}:: \\
\text { NS }: \text { OT-CT }\end{array}$ \\
\hline 6 & $\begin{array}{l}\text { Modified interpretation of modified } \\
\text { sign of } \mathrm{NS}_{\mathrm{x}} \text { in } \mathrm{NS}_{\mathrm{y}}\end{array}$ & $\begin{array}{l}\text { MD-IID }=\mathrm{NS}_{\mathrm{x}}: \mathrm{OT}-\mathrm{CT}:: \\
\text { NS }: \text { OT-CT }\end{array}$ \\
\hline
\end{tabular}

Theoretically speaking, an MD-IID exists in a space of binary time and binary space, such that:

$$
\mathrm{MD}-\mathrm{IID}=\mathrm{S}_{\mathrm{C}}\left(\sum \mathrm{S}_{\mathrm{L}}\left(\mathrm{X}, \mathrm{Y}, \mathrm{Z}, \mathrm{T}_{\mathrm{L}}\right), \mathrm{T}_{\mathrm{C}}\right)
$$

where many four-dimensional local spaces $\mathrm{S}_{\mathrm{L}}$ independently behave within a larger common space $S_{C}$ governed by a common time $\mathrm{T}_{\mathrm{C}}$. It is five-dimensional without knowing how large $\mathrm{S}_{\mathrm{C}}$ is. Table 1 Form 1-6 does not show $\mathrm{T}_{\mathrm{C}}$ and $\mathrm{S}_{\mathrm{C}}$ since it is not necessary to be presented. The (3.1) emphasizes the relativity between any two $\mathrm{S}_{\mathrm{L}}$. MD-IID Scheme can be illustrated in Figure 4.

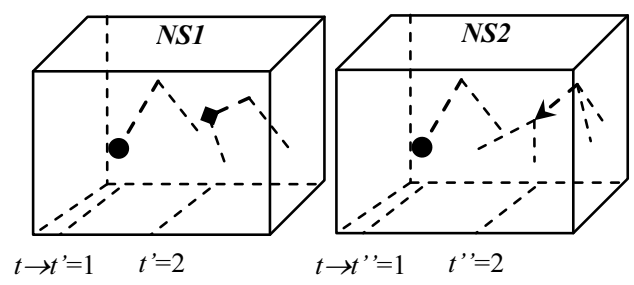

In $\mathrm{t}=1, \mathrm{t}$ ' $=1$ and $\mathrm{t}$ ' $=1$, both NS1 and NS2 has sign $\mathrm{x}$ 's concept $\mathrm{C}=$ “" $\bullet$. However, in $\mathrm{t}$ '=2, NS1 changes $\mathrm{C}=$

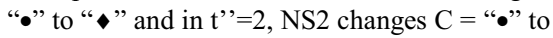
"६". This signifies that in different spaces and their times, the concept of a sign changes differently in a larger space and time. $\left(\mathrm{t} 1=\mathrm{t}^{\prime} 1=\mathrm{t}^{\prime}\right.$ ' $1=\mathrm{OT}$ and $\mathrm{t}^{\prime} 2=\mathrm{CT}$, t'’2 = CT)

Figure 4: Illustration of MD-IID Scheme

MD-IID scheme ensures that any created and modified sign is uniquely identified in spatial and temporal contexts. When a sign is both atomic and independent, called AISign, it is indivisible and can thus be referenced by any other signs without any versioning problem. The philosophy behind the scheme is: anything happened is a history. 
Applying the MD-IID Scheme, the categorized signs can be atomically and independently identified in Table 2 .

Table 2: MD-IID Scheme for All Sign Categories

\begin{tabular}{|c|c|}
\hline Sign Category & Notation \\
\hline Standard sign: $\mathrm{Sign}_{\mathrm{STD}}$ & MD-IID $=$ NS $_{\text {STD }}:$ OT-CT \\
\hline Common sign: Sign $_{\mathrm{COM}}$ & MD-IID $=$ NS $_{\text {STD }}:$ OT-CT $::$ NS $_{\text {COM }}:$ OT-CT \\
\hline Local sign: $\operatorname{Sign}_{\mathrm{LCL}}$ & MD-IID $=$ NS $_{\text {COM }}:$ OT-CT $::$ NS $_{\text {LCL }}:$ OT-CT \\
\hline Personal sign: Sign $_{\mathrm{PNL}}$ & MD-IID = NS PNL $_{2}:$ OT-CT \\
\hline Personal sign cross reference & MD-IID $=$ NS $_{\text {PNL }}:$ OT-CT $::$ NS $_{\text {PNL }}:$ OT-CT \\
\hline
\end{tabular}

From Table 2, we can find that any sign identified by MD-IID is unique in both space and time and no semantic conflict will occur to incur sign versioning problem. This is because each sign only has only one version.

\subsection{Any Social Content Representation}

By atomic and independent signs (AISigns), any social content can be represented (or tagged) without ambiguity and can be generally classified into three types shown in Table 3 as AISign, CSign (composite sign) and DSign (document sign).

Table 3: Types of Signs Representing Social Content

\begin{tabular}{|c|c|}
\hline Type & Definition \\
\hline AISign & AISign $=($ MD-IID $@ X, T$, AN,$<\mathrm{OP}>)$ \\
\hline CSign & $\begin{array}{l}\text { CSign }=\left(\mathrm{AISign}_{1}, \mathrm{AISign}_{2}, \ldots, \mathrm{AISign}_{n}\right), \\
\text { where AISigns are a list such that CSign }=(\mathrm{MD}-\mathrm{IID} @ \mathrm{X},\{\mathrm{T}\} \text {, } \\
\{\mathrm{RT}\},<\mathrm{OP}>) \text { in which MD-IID uniquely identifies the current } \\
\text { AISign, }\{\mathrm{T}\} \equiv\{\mathrm{RT}\} \text { in semantics. Each } \mathrm{rt} \in \mathrm{RT} \text { is an MD-IID } \\
\text { referencing to an external AISign of a vocabulary. }\end{array}$ \\
\hline DSign & $\begin{array}{l}\mathrm{DSign}=\left(\mathrm{AISign}_{1}{ }^{1}, \mathrm{AISign}_{i}{ }^{2}, \ldots, \mathrm{AISign}_{i}{ }^{k}, \ldots, \mathrm{AISign}_{i}{ }^{n}\right), \\
\text { where AISigns form a sign tree with AISign }{ }_{1}^{1} \text { is the tree root, } k \\
\text { is tree level, and } i \text { is sibling position. Alternatively, DSign }= \\
(\mathrm{MD}-\mathrm{IID} @ \mathrm{X},\{\mathrm{T}\},\{\mathrm{RT}\}, \mathrm{PID}, \mathrm{G},<\mathrm{OP}>) \text { in which MD-IID } @ \mathrm{XX} \\
\text { uniquely identifies the current AISign, }\{\mathrm{T}\} \equiv\{\mathrm{RT}\} \text { pointing to } \\
\text { external AISigns of vocabularies, PID is a parent AISign MD- } \\
\text { IID, and G defines the grammar relationship between the cur- } \\
\text { rent AISign and children AISigns. }\end{array}$ \\
\hline
\end{tabular}

The signs described in Table 3 are used to represent social content. For AISign, X of MD-IID likes a dictionary name (or its address) and MD-IID likes a word or a fixed phrase in the linguistic form of $\mathrm{T}$ and with the sense of AN. For CSign, $\mathrm{X}$ of MD-IID likes namespace where MD-IID is defined for a new AISign that is further defined by a sequence of AISigns. The MD-IID uses $\{\mathrm{T}\}$ and $\{\mathrm{RT}\}$ to define a non-fixed phrase, word-phrase combinations, sentences or even a paragraph as long as the MD-IID denotes a sequence of external AISigns to express a new meaning. For DSign, $\mathrm{X}$ of MD-IID refers to the current document namespace where MD-IID is defined for a new AISign that is further defined by sub-tree of AISigns. The MD-IID uses $\{\mathrm{T}\},\{\mathrm{RT}\}$ and $\mathrm{G}$ to define a sub-tree of CSigns and applies PID to notate the current position of the defining AISign. In Sign Network, G of DSign can define how the current AISign introduces a type of sub-document in grammar, as shown in Table 4.
Table 4: Sub-Document Introduced by G Parameter

\begin{tabular}{|c|c|}
\hline Value of G & Sub-Document Introduced \\
\hline $\mathrm{G}=\mathrm{T}$ & Term \\
\hline $\mathrm{G}=\mathrm{H}$ & Non-fixed phrase \\
\hline $\mathrm{G}=\mathrm{S}$ & Sentence \\
\hline $\mathrm{G}=\mathrm{P}$ & Paragraph \\
\hline $\mathrm{G}=\mathrm{C}$ & Section \\
\hline $\mathrm{G}=\mathrm{B}$ & Table \\
\hline $\mathrm{G}=\mathrm{F}$ & Figure \\
\hline
\end{tabular}

In Sign Network, a document sign is recursively developed by the represented content using two technologies of concept-based input method and concept-oriented grammar detection method. The concept-based input method specifies that any source of input is AISigns from different vocabularies connected to the document editor. This is different from the traditional input, which is from the existing characters like English letters. The concept-oriented grammar detection method states that any inputted AISign will be checked together with the previous-already content to determine the new grammar structure of the whole document. Technically, its procedure is designed as Figure 5:

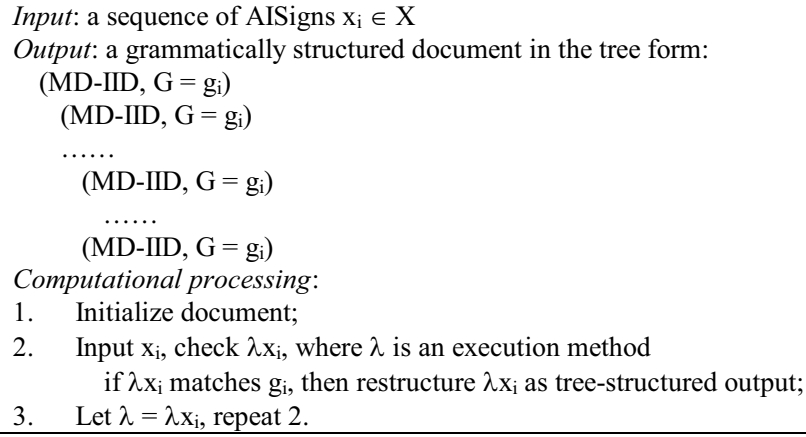

Figure 5: Concept-oriented grammar detection

Applying the above two methods, any social content can be semantically represented in DSign where any AISign is a sub-document of DSign and can be independently used in any context.

\section{Sign-based Social Computing}

To semantically enable social computing for social navigation, social tagging and social collaboration, this Section introduces a sign-based social computing technique, called Sign Exchange Mechanism, and applies it to tag and navigate social content for collaboration.

\subsection{Sign Exchange Mechanism}

Applying the sign-based semantic representation described previously, a Sign Exchange Mechanism is designed. In Figure 6, Sign Exchange Mechanism consists of a Common AISign Localizer (CAL) to edit local AISigns in $\mathrm{V}_{\mathrm{L}}$, a Personal AISign Editor (PAE) to edit personal AISigns in $V_{P}$, a Personal AISign Interface (PSI) to allow personal AISigns used in the exchanged DSign to reference the original senses of $V_{P}$, a CSign Generator (CSignG) to create composite signs (CSigns) of a local node domain from $\mathrm{V}_{\mathrm{L}}$ 
and $V_{\mathrm{P}}$, a DSign Generator (DSignG) to create document signs (DSigns) of a local node domain from $\mathrm{V}_{\mathrm{L}}, \mathrm{V}_{\mathrm{P}}$, and a DSign Exchanger (DSignE) to exchange social content between local nodes.

In this mechanism, any DSign is concealed and interfaceable, that is, any sub-tree of a DSign is a semantic unit or a concept that is uniquely understandable by any external nodes. This feature makes any heterogeneous social contents semantically interoperable.

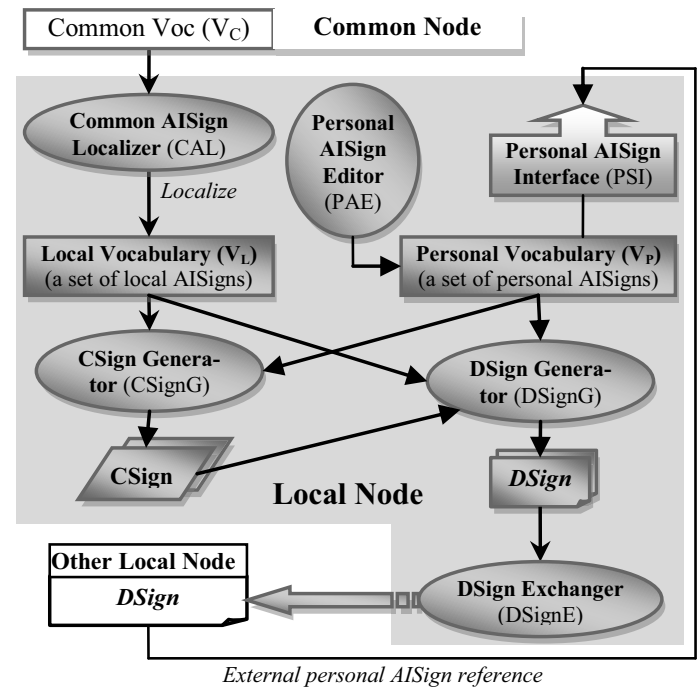

Figure 6: Sign Exchange Mechanism

\subsection{Sign-based Social Computing Applications}

In this Section, we present three sign-based semanticsenriched social computing applications to illustrate how Sign Exchange Mechanism helps social computing in heterogeneous environment for social tagging, social navigation and social collaboration. In social tagging, to tag Web resources such as songs and favorites, we simply tag each of them as a CSign as a sequence of local or personal AISigns. In social navigation, we navigate the social content tagged as CSigns following the NS of MD-IID of tagged social content. For social collaboration, in order for any Web users to collaborate, we represent collaborative content as DSigns by using different ASigns of $\mathrm{V}_{\mathrm{S}}, \mathrm{V}_{\mathrm{C}}, \mathrm{V}_{\mathrm{L}}$ and $\mathrm{V}_{\mathrm{P}}$. After the DSigns are received by the receiver, they are interpreted by checking MD-IID referenced in different NS for $\mathrm{V}_{\mathrm{S}}, \mathrm{V}_{\mathrm{C}}, \mathrm{V}_{\mathrm{L}}$ and $\mathrm{V}_{\mathrm{P}}$. Figure 7 illustrates the design of these applications.

In Figure 7, the result of tagging is the generation of many personal tags as personal signs, local tags as local signs and common tags as common signs. Tags of others can be further tagged or used by following MD-IID scheme of $\mathrm{NS}_{\mathrm{x}}: \mathrm{OT}-\mathrm{CT}: \mathrm{NS}_{\mathrm{y}}: \mathrm{OT}-\mathrm{CT}$. These created tags can be accurately navigated by others following their attached NS in NS:OT-CT and can be traced back to the first one who originally tag the social content. Sign Exchange Mechanism can design and use any contents by strategies of atomicity, independence, uniqueness, concealment and interface.
These strategies make the content semantically interoperable between Web users.

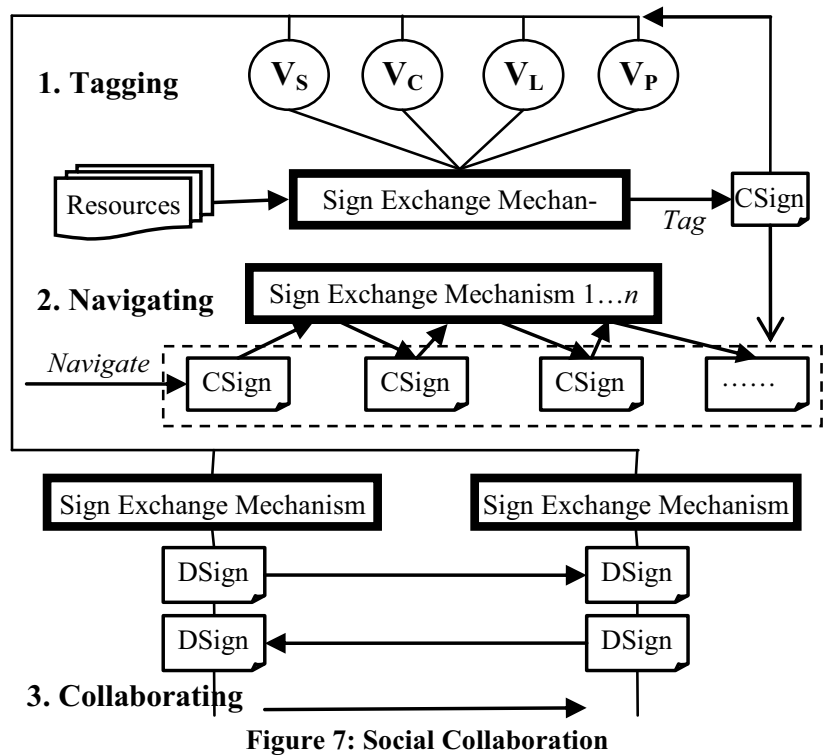

\subsection{An Integrated Example}

To better understand Sign Network and Sign Exchange Mechanism, we present an integrated example of collaborative tagging for automatic navigation and collaborative ebusiness, shown in Figure 8, 9, 10 and 11.

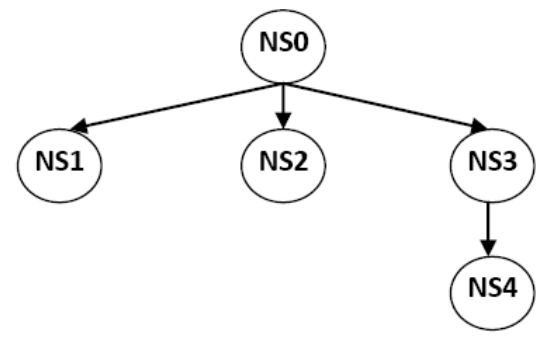

Figure 8: Example nodes of a sign network

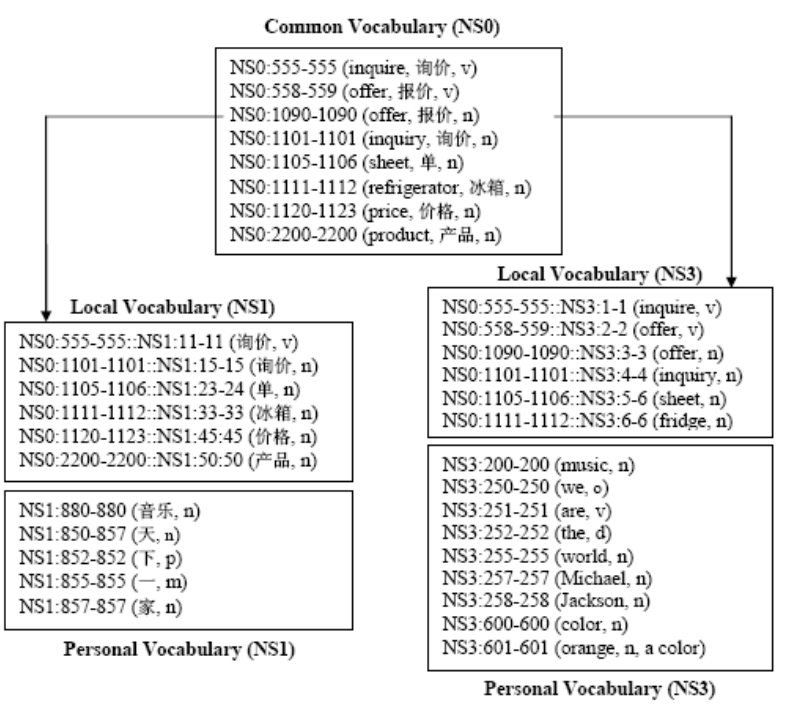

Figure 9: Example data of a node in sign network 
Figure 8 shows a simple sign network consists of one common node NS0, three local nodes NS1, NS2 and NS3 of the common node NS0 and one local node NS4 of a local node NS3. The Figure 9 shows that common node NS0 has a common vocabulary sharable by all local nodes NS1, NS2 and NS3. The local nodes NS1 and NS3 both have their local vocabularies and personal vocabularies. A term in a local vocabulary is transformable to a term of a common vocabulary since its identifier MD-IID includes the identifier specified in a common term. A personal vocabulary is concealed and understandable by namespace reference.

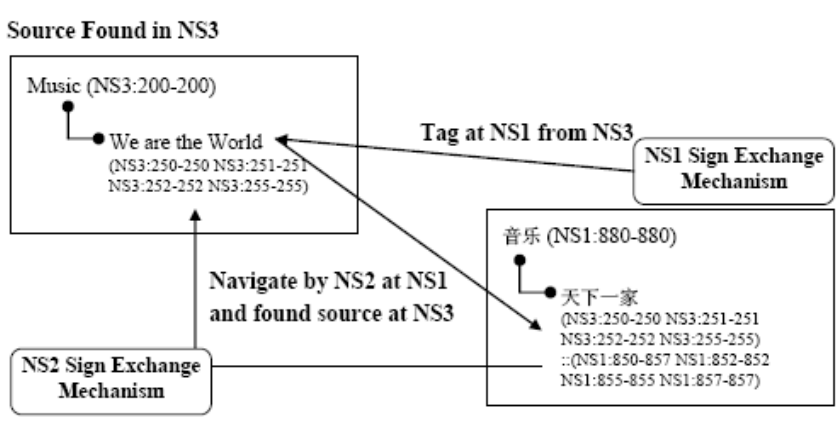

Figure 10: Collaborative tagging and navigation

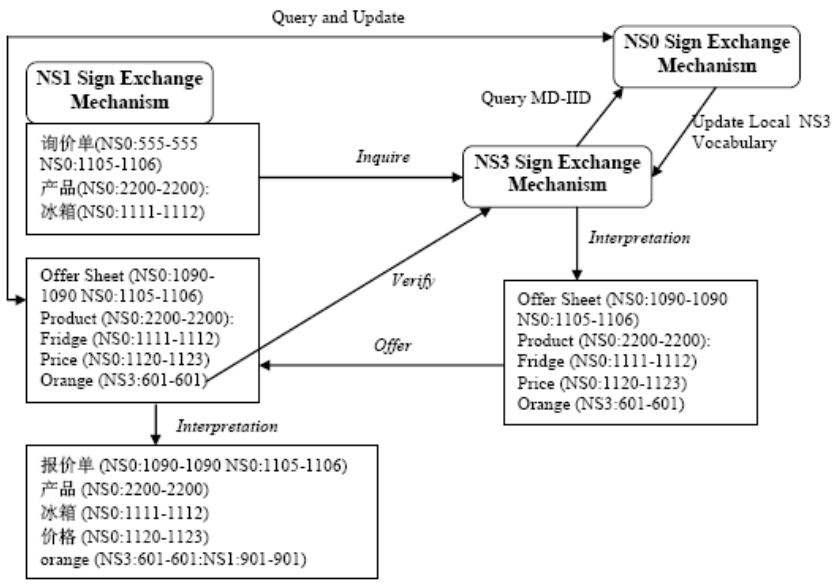

Figure 11: Collaborative e-business

Common, local and personal vocabularies can be used to design applications of tagging, navigation and collaborative work. For example, in Figure 9, the entity NS1 and NS3 both have a personal vocabulary containing their personal AISigns. In Figure 10, NS3 maintains a music directory including a song "We are the World", which is tagged using NS3's AISigns in English as NS3:250-250 NS3:251-251 NS3:252-252 NS3:255-255. A user in NS1 has found the song and further tags it in Chinese as (NS3:250-250 NS3:251-251 NS3:252-252 NS3:255-255)::(NS1:850-857 NS1:852-852 NS1:855-855 NS1:857-857). This is a typical direct term reference. Another user in NS2 navigates the song from Chinese listing in NS1 to NS3 to finally find downloadable sources. It should be noted that in implementation finding the source by navigation can be automatic.
Besides the collaborative tagging for automatic navigation, Figure 11 shows a process of doing e-business through collaborative work. NS1 issues a 询价单 (inquiry sheet) to NS3. NS3 finds terms are understandable through MD-IID of common vocabulary. It then interprets it and makes an offer to NS1. In this offer, some terms are personal terms of NS3 (e.g., orange). When the offer is received by NS1, NS1 interprets the offer using MD-IID scheme. When encountering "orange", it adds the right part of the MD-IID like create a new tag.

The examples illustrate an accurate and universal tagging scheme, which can be applied to accurate navigation and e-business collaborative work.

\section{Conclusion}

Sign-based semantics-enriched social computing allows the users of Social Web to tag, navigate, and collaborate in a larger community without meaning ambiguity during their interaction. The research on this track will enable the present Social Web to be successfully transformed towards the future Semantic Social Web that benefits most of the existing Web users.

Sign Network research described in this paper is an attempt of adding semantics to the existing social computing. It is a novel approach to the semantic interoperability challenge that faces semantic technology and Social Web.

Sign Network represents semantics based on the theory of semiotics and the prior work of collaborative conceptualization. It represents semantics on socially and collaboratively developed signs, which are subjective interpretation of any physical, conceptual or fictitious objects of realworld reality. Technically, sign is a construct of structure, concept, context and interpretant. It states that any sign is a personal interpretation of an interpretant at his/her own context and this interpretation is conceptualized as a written term that is further uniquely identified in the spatial and temporal context of the interpretant. In terms of concept, each sign is atomic and independent (AISign), which can be referenced anywhere without meaning ambiguity to formulate composite signs (CSign) and document sign (DSign).

Sign Network is an important contribution to the semantics technology and social computing. It provides a rather comprehensive solution to semantics interoperability for sign exchange by proposing a series of methods such as sign-based representation, composition and exchange by MD-IID scheme. As an artificial sign language, Sign Network is different from the existing ontology language, such as OWL (w3.org/TR/owl-features) that is only a semantics format language.

Sign Network research is still evolving. Currently, a large sign database in real has already been implemented in University of Macau, where English is used as a standard language to bridge the senses of several English and Chinese dictionaries. Some collaborative designers are map- 
ping these dictionaries onto the synonymous senses given by English WordNet (wordnet.princeton.edu). In the implementation, UNSPSC (unspsc.org) is modified to provide the high-level term categorization and some other international standards on measurement, unit, and domain vocabularies are also under mapping.

In future, we will launch a public Website to provide the open access to our implemented sign database. So, Social Web users can be leveraged to tag, navigate and collaborate together.

\section{Acknowledgement}

This research is partially supported by University of Macau Research Grant No. RG055/08-09S/GJZ/FST and RG055/08-09S/10R/GJZ/FST.

\section{References}

[1] Aristotle. Metaphysics. Translated by W. Ross, 2007. http://ebooks.adelaide.edu.au/a/aristotle/metaphysics/.

[2] Barthes, R., Mythologies, Hill and Wang, 1972.

[3] Bi, B., Shang, L. and B. Kao (2009) Collaborative Resource Discovery in Social Tagging Systems. In: Proc. of $A C M$ CIKM'09, pp. 1919-1922.

[4] Cheok, A., Yang, X., Ying, Z., Billinghurst, M. and H. Kato (2002) Touch-Space: Mixed Reality Game Space Based on Ubiquitous, Tangible, and Social Computing. Personal and Ubiquitous Computing 6(5-6) 430-442.

[5] Dijk, T. (1977) Text and context: Explorations in the Semantics and Pragmatics of Discourse. Longman.

[6] Fischer, G., Resnick, M., Jennings, P., Shneiderman, B. and M. Maher (2009) Creativity Challenges and Opportunities in Social Computing. In: ACM CHI 2009 (April 4-9, Boston, USA) pp. 3283-3286.

[7] Franssila, H. and P. Mannonen (2009) Understanding social entities, activity coupling and social computing affordances in industrial work environments. In: ECCE'09 (Helsinki, Finland) VTT Technical Research Centre of Finland.

[8] Gomes, A., Gudwin, R. and J. Queiroz (2003) Towards Meaning Processes in Computers from Peircean Semiotics. S.E.E.D. Journal (Semiotics, Evolution, Energy, and Development) 3(2):69-79.

[9] Gruber, T. R. (1993) A translation approach to portable ontologies. Knowledge Acquisition 5(2) 199-220.

[10]Guo, J. (2008) Collaborative Concept Exchange. VDM Verlag, Germany.

[11] Guo, J. (2009) Collaborative Conceptualization: Towards a Conceptual Foundation of Interoperable Electronic Product Catalogue System Design. Enterprise Information Systems 3(1):59-94.
[12] Hepp, M., Siorpaes, K. and D. Bachlechner (2007) Harvesting Wiki Consensus: Using Wikipedia Entries as Vocabulary for Knowledge Management. IEEE Internet Computing 11(5):54-65.

[13] Hsieh, W., Stu, J., Chen, Y. and S. Chou (2009) A collaborative desktop tagging system for group knowledge management based on concept space. Expert Systems with Applications 36:9513-9523.

[14] Huang, A. and T. Chuang (2009) Social tagging, online communication, and Peircean semiotics: a conceptual framework. Journal of Information Science 35(3):340 357.

[15] King, I., Li, J. and K. Chan (2009) A brief survey of computational approaches in social computing. In: IEEE Proc. of IJCNN'09, pp. 1625-1632.

[16] Millen, D., Feinberg, J. and B. Kerr (2006) Dogear: Social Bookmarking in the Enterprise. In: $A C M C H I$ 2006 Proceedings, pp. 111-120.

[17] Peirce, C. (1839-1914). Peirce on Signs: Writings on Semiotics, edited by James Hoops. The University of North Carolina Press (1991).

[18] Saussure, F., 1966. Course in general linguistics. New York: McGraw-Hill Book Company.

[19] Schenkel, R., Crecelius, T., Kacimi, M., Michel, S., Neumann, T., Parreira, J. and G. Weikum (2008) Efficient Top-k Querying over Social-Tagging Networks. In: Proc. of ACM SIGIR'08, pp. 523-530.

[20] Sheth, A. and M. Nagarajan (2009) SemanticsEmpowered Social Computing. IEEE Internet Computing 13(1): 76-80.

[21] Schuler, D. (1994) Social Computing. Communications of the ACM 37(1) 28-29.

[22] Soriano, J., López, J., Jiménez, M. and F. Alonso (2008) Enabling Semantics-Aware Collaborative Tagging and Social Search in an Open Interoperable Tagosphere. In: ACM Proc. of iiWAS2008, pp. 84-91.

[23] Storey, M., Cheng, L., Bull, I. and P. Rigby (2006) Shared Waypoints and Social Tagging to Support Collaboration in Software Development. In: Proc. of ACM CSCW'06, pp. 195-198.

[24] Subramanya, S. and H. Liu (2008) SocialTagger - Collaborative Tagging for Blogs in the Long Tail. In: Proc. of ACM Workshop on Search in Social Media, pp. 19-26.

[25] Wang, F., Carley, K., Zeng, D. and W. Mao (2007) Social Computing: From Social Informatics to Social Intelligence. IEEE Intelligent Systems 22(2) 79-83.

[26] Yeung, C., Gibbins, N. and N. Shadbolt (2009) Contextualising Tags in Collaborative Tagging Systems. In: Proc. of ACM HT'09, pp. 251-260. 